This is an electronic reprint of the original article. This reprint may differ from the original in pagination and typographic detail.

Author(s): Anjam, Immanuel; Nokka, Marjaana; Repin, Sergey

Title: $\quad$ On a posteriori error bounds for approximations of the generalized Stokes problem generated by the Uzawa algorithm

Year: $\quad 2012$

Version:

Please cite the original version:

Anjam, I., Nokka, M., \& Repin, S. (2012). On a posteriori error bounds for approximations of the generalized Stokes problem generated by the Uzawa algorithm. Russian Journal of Numerical Analysis and Mathematical Modelling, 27(4), 321-338. https://doi.org/10.1515/rnam-2012-0018

All material supplied via JYX is protected by copyright and other intellectual property rights, and duplication or sale of all or part of any of the repository collections is not permitted, except that material may be duplicated by you for your research use or educational purposes in electronic or print form. You must obtain permission for any other use. Electronic or print copies may not be offered, whether for sale or otherwise to anyone who is not an authorised user. 


\title{
On a posteriori error bounds for approximations of the generalized Stokes problem generated by the Uzawa algorithm
}

\author{
I. ANJAM, M. NOKKA ${ }^{\dagger}$ and S.I. REPIN $\$$
}

Abstract - In this paper, we derive computable a posteriori error bounds for approximations computed by the Uzawa algorithm for the generalized Stokes problem. We show that for each Uzawa iteration both the velocity error and the pressure error are bounded from above by a constant multiplied by the $L_{2}$-norm of the divergence of the velocity. The derivation of the estimates essentially uses a posteriori estimates of the functional type for the Stokes problem.

\section{Introduction}

Let $\Omega \in \mathbb{R}^{n}$ be a bounded connected domain with a Lipschitz continuous boundary $\partial \Omega$. Henceforth, we use the space of vector valued functions

$$
V\left(\Omega, \mathbb{R}^{n}\right):=W_{2}^{1}\left(\Omega, \mathbb{R}^{n}\right)
$$

and two spaces of tensor-valued functions

$$
\begin{aligned}
\Sigma(\Omega) & :=L_{2}\left(\Omega, \mathbb{M}^{n \times n}\right) \\
\Sigma(\operatorname{Div}, \Omega) & :=\left\{w \in \Sigma(\Omega) \mid \operatorname{Div} w \in L_{2}\left(\Omega, \mathbb{R}^{n}\right)\right\}
\end{aligned}
$$

where $\mathbb{M}^{n \times n}$ is the space of symmetric $n \times n$-matrices (tensors). The scalar product of tensors is denoted by two dots (:), and the $L_{2}$ norm of $\Sigma$ is denoted by $\|\cdot\|_{\Sigma}$. The $L_{2}$ norm of scalar and vector valued functions is denoted by $\|\cdot\|$.

By $\stackrel{\circ}{S}(\Omega)$ we denote the closure of smooth solenoidal functions $w$ with compact supports in $\Omega$ with respect to the norm $\|\nabla w\|_{\Sigma}$. Let $V_{0}\left(\Omega, \mathbb{R}^{n}\right)$ denote the subspace

\footnotetext{
* University of Jyväskylä, Department of Mathematical Information Technology, P.O. Box 35 (Agora), FI-40014 University of Jyväskylä, Finland. Email: immanuel.anjam@jyu.fi

$\dagger$ University of Jyväskylä, Department of Mathematical Information Technology, P.O. Box 35 (Agora), FI-40014 University of Jyväskylä, Finland. Email: marjaana.nokka@jyu.fi

¥V. A. Steklov Institute of Mathematics in St. Petersburg, Fontanka 27, RU-191024, St. Petersburg, Russia. Email: repin@pdmi.ras.ru

This work was supported by the Finnish foundation Emil Aaltosen säätiö and Russian Foundation for Basic Research Grants 11-01-00531a, 11-01-00324a.
} 
of $V\left(\Omega, \mathbb{R}^{n}\right)$ that consists of functions with zero traces on $\partial \Omega$. The space of scalar valued square summable functions with zero mean is denoted by $\widetilde{L}_{2}(\Omega, \mathbb{R})$.

The classical statement of the generalized Stokes problem consists of finding a velocity field $u \in \stackrel{S}{S}(\Omega)+u_{D}$ and pressure $p \in \widetilde{L}_{2}(\Omega)$ which satisfy the relations

$$
\begin{aligned}
-\operatorname{Div}(v \nabla u)+\mu u+\nabla p=f & \text { in } \Omega \\
\operatorname{div} u=0 & \text { in } \Omega \\
u=u_{D} & \text { on } \partial \Omega
\end{aligned}
$$

where $f \in L_{2}\left(\Omega, \mathbb{R}^{n}\right)$, and

$$
\int_{\partial \Omega} u_{D} \cdot n \mathrm{~d} x=0
$$

Here and later on $n$ denotes the outward unit normal vector to $\partial \Omega$, and we assume that the material parameters $v$ and $\mu$ belong to the space $L_{\infty}(\Omega, \mathbb{R})$, and

$$
\begin{array}{ll}
0<\underline{v} \leqslant v(x) \leqslant \bar{v}, & \forall x \in \bar{\Omega} \\
0 \leqslant \underline{\mu} \leqslant \mu(x) \leqslant \bar{\mu}, & \forall x \in \bar{\Omega} .
\end{array}
$$

The generalized solution of (1.1)-(1.3) is a function $u \in \stackrel{S}{(\Omega)}\left(\Omega u_{D}\right.$ such that

$$
\int_{\Omega}(v \nabla u: \nabla w+\mu u \cdot w) \mathrm{d} x=\int_{\Omega} f \cdot w \mathrm{~d} x \quad \forall w \in \stackrel{\circ}{(}(\Omega) .
$$

It is well known that $u$ can be defined as the first component of the saddle point problem generated by any of the Lagrangians

$$
\begin{aligned}
L(v, q) & :=\int_{\Omega}\left(\frac{1}{2} v|\nabla v|^{2}+\frac{1}{2} \mu|v|^{2}-q \operatorname{div} v-f \cdot v\right) \mathrm{d} x \\
L_{A}(v, q) & :=\int_{\Omega}\left(\frac{1}{2} v|\nabla v|^{2}+\frac{1}{2} \mu|v|^{2}+\frac{1}{2} \lambda|\operatorname{div} v|^{2}-q \operatorname{div} v-f \cdot v\right) \mathrm{d} x .
\end{aligned}
$$

The quantity in $L_{A}$ is called the augmented Lagrangian (in which $\lambda \in \mathbb{R}_{+}$). We have

$$
\begin{gathered}
L(v, p) \leqslant L(u, p) \leqslant L(u, q) \quad \forall v \in V_{0}+u_{D}, q \in L_{2} \\
L_{A}(v, p) \leqslant L_{A}(u, p) \leqslant L_{A}(u, q) \quad \forall v \in V_{0}+u_{D}, q \in L_{2} .
\end{gathered}
$$

From the right-hand side inequalities we see that $\int_{\Omega}(p-q) \operatorname{div} u \mathrm{~d} x=0$ for all $q \in L_{2}$, from which we conclude that $\operatorname{div} u=0$. From the left-hand side inequalities it follows that for any solenoidal $v$ we have $J(v)>J(u)$, where

$$
J(v):=\int_{\Omega}\left(\frac{1}{2} v|\nabla v|^{2}+\frac{1}{2} \mu|v|^{2}-f \cdot v\right) \mathrm{d} x .
$$


Indeed, the exact solution of the problems

$$
\inf _{v \in V_{0}+u_{D}} \sup _{q \in L_{2}} L(v, q), \quad \inf _{v \in V_{0}+u_{D}} \sup _{q \in L_{2}} L_{A}(v, q)
$$

is $(u, p)$. For a detailed exposition of this subject, we refer to [4].

Finding approximations of $(u, p)$ can be performed by the Uzawa algorithm presented below.

\section{Algorithm 1.1 (Uzawa algorithm).}

1: Set $\mathrm{k}=0$ and $\rho \in \mathbb{R}_{+}$. Make initial guess for $p^{k} \in \widetilde{L}_{2}$.

2: Find $u^{k}$ by minimizing the Lagrangian $L\left(v, p^{k}\right)$ or $L_{A}\left(v, p^{k}\right)$ w.r.t. $v$, i.e., by solving either (1.5) or (1.6), respectively.

For the Lagrangian $L$, we have the problem: Find $u^{k} \in V_{0}+u_{D}$ such that:

$$
\int_{\Omega}\left(v \nabla u^{k}: \nabla w+\mu u^{k} \cdot w\right) \mathrm{d} x=\int_{\Omega}\left(f \cdot w+p^{k} \operatorname{div} w\right) \mathrm{d} x \quad \forall w \in V_{0} .
$$

For the Lagrangian $L_{A}$, we have the problem: Find $u^{k} \in V_{0}+u_{D}$ such that:

$$
\begin{aligned}
& \int_{\Omega}\left(v \nabla u^{k}: \nabla w+\mu u^{k} \cdot w+\lambda \operatorname{div} u^{k} \operatorname{div} w\right) \mathrm{d} x \\
&=\int_{\Omega}\left(f \cdot w+p^{k} \operatorname{div} w\right) \mathrm{d} x \quad \forall w \in V_{0} .
\end{aligned}
$$

3: Find

$$
p^{k+1}=\left(p^{k}-\rho \operatorname{div} u^{k}\right) \in \widetilde{L}_{2} .
$$

4: Set $k=k+1$ and go to step 2 .

Our goal is to deduce computable bounds of the difference between $u^{k}$ and the exact solution $u$ in terms of the energy norms

$$
\|w\|^{2}:=\int_{\Omega}\left(v|\nabla w|^{2}+\mu|w|^{2}\right) \mathrm{d} x
$$

and

$$
]\left.\|w\|\right|_{\lambda} ^{2}:=\int_{\Omega}\left(v|\nabla w|^{2}+\mu|w|^{2}+\lambda|\operatorname{div} w|^{2}\right) \mathrm{d} x .
$$

Theorem 1.1. The Uzawa algorithm (Algorithm 1.1) converges, i.e.,

$$
\begin{array}{ll}
u^{k} \stackrel{k \rightarrow \infty}{\longrightarrow} u & \text { strongly in } V\left(\Omega, \mathbb{R}^{n}\right) \\
p^{k} \stackrel{k \rightarrow \infty}{\longrightarrow} p & \text { weakly in } L_{2}(\Omega)
\end{array}
$$

provided that

$$
0<\rho<2 \min (\underline{v}, \underline{\mu})
$$

and $p^{0} \in \widetilde{L}_{2}(\Omega)$. If $\mu \equiv 0$, the condition is $0<\rho<2 \underline{v}$. These conditions are the same for both (1.5) and (1.6). 
Proof. The proof is based on well known arguments (see, e.g., [13]). However, for the convenience of the reader, we present the proof for the generalized Stokes problem, in the case of (1.5).

The exact solution of the generalized Stokes problem satisfies the relation

$$
\int_{\Omega}(v \nabla u: \nabla w+\mu u \cdot w) \mathrm{d} x=\int_{\Omega}(f \cdot w+p \operatorname{div} w) \mathrm{d} x \quad \forall w \in V_{0}(\Omega) .
$$

We set $w=u^{k}-u$ and subtract (1.9) from (1.5), which gives

$$
\left\|u^{k}-u\right\|^{2}=\int_{\Omega}\left(p^{k}-p\right) \operatorname{div}\left(u^{k}-u\right) \mathrm{d} x
$$

Let $v^{k}:=u^{k}-u$ and $q^{k}:=p^{k}-p$. Then we rewrite this relation in the form

$$
\left\|v^{k}\right\|^{2}=\int_{\Omega} q^{k} \operatorname{div} v^{k} \mathrm{~d} x
$$

On the other hand, (1.7) is equivalent to

$$
\int_{\Omega}\left(p^{k+1}-p^{k}\right) \phi \mathrm{d} x+\rho \int_{\Omega} \operatorname{div} u^{k} \phi \mathrm{d} x=0 \quad \forall \phi \in L_{2}(\Omega) .
$$

By setting $\phi=p^{k+1}-p$ we obtain

$$
\int_{\Omega}\left(p^{k+1}-p^{k}\right)\left(p^{k+1}-p\right) \mathrm{d} x+\rho \int_{\Omega} \operatorname{div} u^{k}\left(p^{k+1}-p\right) \mathrm{d} x=0
$$

which is equivalent to

$$
\int_{\Omega}\left(q^{k+1}-q^{k}\right) q^{k+1} \mathrm{~d} x+\rho \int_{\Omega} \operatorname{div} v^{k} q^{k+1} \mathrm{~d} x=0
$$

and

$$
\left\|q^{k+1}\right\|^{2}-\left\|q^{k}\right\|^{2}+\left\|q^{k+1}-q^{k}\right\|^{2}=-2 \rho \int_{\Omega} \operatorname{div} v^{k} q^{k+1} \mathrm{~d} x .
$$

By combining (1.10) and (1.11), we obtain

$$
\begin{aligned}
\left\|q^{k+1}\right\|^{2}-\left\|q^{k}\right\|^{2}+ & \left\|q^{k+1}-q^{k}\right\|^{2}+2 \rho\left\|v^{k}\right\| \\
& =-2 \rho \int_{\Omega} \operatorname{div} v^{k}\left(q^{k+1}-q^{k}\right) \mathrm{d} x \\
& \leqslant 2 \rho\left\|\operatorname{div} v^{k}\right\|\left\|q^{k+1}-q^{k}\right\| \\
& \leqslant \delta^{-1} \rho^{2}\left\|\operatorname{div} v^{k}\right\|^{2}+\delta\left\|q^{k+1}-q^{k}\right\|^{2} \\
& \leqslant \delta^{-1} \rho^{2}\left(\left\|\nabla v^{k}\right\|_{\Sigma}^{2}+\left\|v^{k}\right\|^{2}\right)+\delta\left\|q^{k+1}-q^{k}\right\|^{2}
\end{aligned}
$$


where $\delta \in(0,1)$. Note that

$$
\left\|v^{k}\right\|^{2} \geqslant \underline{v}\left\|\nabla v^{k}\right\|_{\Sigma}^{2}+\underline{\mu}\left\|v^{k}\right\|^{2} \geqslant \min (\underline{\nu}, \underline{\mu})\left(\left\|\nabla v^{k}\right\|_{\Sigma}^{2}+\left\|v^{k}\right\|^{2}\right)
$$

and, therefore, (1.12) implies the estimates

$$
\begin{aligned}
\left\|q^{k+1}\right\|^{2}-\left\|q^{k}\right\|^{2} & +(1-\delta)\left\|q^{k+1}-q^{k}\right\|^{2} \\
& +\rho\left(2 \min (\underline{\nu}, \underline{\mu})-\delta^{-1} \rho\right)\left(\left\|\nabla v^{k}\right\|_{\Sigma}^{2}+\left\|v^{k}\right\|^{2}\right) \leqslant 0 .
\end{aligned}
$$

Now, we sum inequalities (1.13) for $k=0, \ldots, N$ and find that

$$
\begin{aligned}
\left\|q^{N+1}\right\|^{2} & +(1-\delta) \sum_{k=0}^{N}\left\|q^{k+1}-q^{k}\right\|^{2} \\
& +\rho\left(2 \min (\underline{\nu}, \underline{\mu})-\delta^{-1} \rho\right) \sum_{k=0}^{N}\left(\left\|\nabla v^{k}\right\|_{\Sigma}^{2}+\left\|v^{k}\right\|^{2}\right) \leqslant\left\|q^{0}\right\| .
\end{aligned}
$$

Because of condition (1.8), there exists a $\delta_{*} \in(0,1)$ such that

$$
2 \min (\underline{v}, \underline{\mu})-\delta_{*}^{-1} \rho>0 .
$$

We set $\delta=\delta_{*}$ in (1.14), and see that

$$
\left\|\nabla v^{k}\right\|_{\Sigma}^{2}+\left\|v^{k}\right\|^{2}=\left\|\nabla\left(u^{k}-u\right)\right\|_{\Sigma}^{2}+\left\|u^{k}-u\right\|^{2} \stackrel{k \rightarrow \infty}{\longrightarrow} 0 .
$$

Also, we see that $\left\|q^{k}\right\|=\left\|p^{k}-p\right\|$ is bounded in $L_{2}(\Omega)$, so $\left\|p^{k}\right\|$ is bounded in $L_{2}(\Omega)$. We also observe from (1.14), that

$$
\left\|q^{k+1}-q^{k}\right\|^{2}=\left\|p^{k+1}-p^{k}\right\|^{2} \stackrel{k \rightarrow \infty}{\longrightarrow} 0
$$

so we can extract from $p^{k}$ a subsequence $p^{k^{\prime}}$, which converges to some element $p^{*}$ weakly in $L_{2}(\Omega)$. The equation (1.5) gives in the limit

$$
\int_{\Omega}(v \nabla u: \nabla w+\mu u \cdot w) \mathrm{d} x=\int_{\Omega}\left(f \cdot w+p^{*} \operatorname{div} w\right) \mathrm{d} x \quad \forall w \in V_{0}
$$

and by comparison to (1.9) we find that

$$
\int_{\Omega}\left(p-p^{*}\right) \operatorname{div} w \mathrm{~d} x=0 \quad \forall w \in V_{0}
$$

which means that $p^{*}=p+c$, where $c \in \mathbb{R}$. In other words, the sequence $p^{k^{\prime}}$ converges weakly to $p$ in $\widetilde{L}_{2}(\Omega)$ However, if $p^{0} \in \widetilde{L}_{2}$, then it is easy to see from (1.7) that $p^{k} \in \widetilde{L}_{2}$ with all $k$. From this we make the conclusion that the sequence $p^{k^{\prime}}$ converges weakly to $p$ in $L_{2}(\Omega)$. 


\section{Error estimates for exact solutions generated by the Uzawa algorithm}

In this section, we show that the errors of approximations generated by the Uzawa algorithm are controlled by the $L_{2}$-norm of the divergence of the velocity. First, we compare approximations computed on two consequent iterations and establish the following result.

Theorem 2.1. Let $\left(u^{k}, p^{k}\right)$ and $\left(u^{k+1}, p^{k+1}\right)$ be the solutions of two consecutive iterations of the Uzawa algorithm. Then, for both (1.5) and (1.6) we have

$$
\begin{aligned}
\left\|u^{k+1}-u^{k}\right\| & \leqslant \sqrt{\underline{\underline{v}}}^{-1} \rho\left\|\operatorname{div} u^{k}\right\| \\
\left\|p^{k+1}-p^{k}\right\| & =\rho\left\|\operatorname{div} u^{k}\right\| .
\end{aligned}
$$

In addition, for (1.6) we also have

$$
]\left\|u^{k+1}-u^{k}\right\|\left[\lambda \leqslant \sqrt{\underline{v}}^{-1} \rho\left\|\operatorname{div} u^{k}\right\| .\right.
$$

Proof. The equation for pressure (2.2) follows directly from (1.7). By subtracting the $k$ th equation $(1.5)$ from the $(k+1)$ th equation, we obtain

$$
\int_{\Omega} v \nabla\left(u^{k+1}-u^{k}\right): \nabla w+\mu\left(u^{k+1}-u^{k}\right) \cdot w \mathrm{~d} x=\int_{\Omega}\left(p^{k+1}-p^{k}\right) \operatorname{div} w \mathrm{~d} x .
$$

Since

$$
\|\operatorname{div} w\| \leqslant\|\nabla w\|_{\Sigma} \leqslant \sqrt{\underline{v}}^{-1}\|\sqrt{v} \nabla w\|_{\Sigma} \leqslant \sqrt{\underline{v}}^{-1} \mid\|w\|
$$

we can estimate the right-hand side with

$$
\begin{aligned}
\int_{\Omega}\left(p^{k+1}-p^{k}\right) \operatorname{div} w \mathrm{~d} x & \leqslant\left\|p^{k+1}-p^{k}\right\|\|\operatorname{div} w\| \\
& \leqslant \sqrt{\underline{\underline{v}}}^{-1}\left\|p^{k+1}-p^{k}\right\|\|w\| .
\end{aligned}
$$

By choosing $w=u^{k+1}-u^{k}$, we obtain

$$
\left\|u^{k+1}-u^{k}\right\|^{2} \leqslant \sqrt{\underline{\underline{v}}}^{-1}\left\|p^{k+1}-p^{k}\right\|\left\|u^{k+1}-u^{k}\right\| .
$$

By (2.2) we obtain the estimate for velocity (2.1). The estimate (2.3) is obtained with exactly the same arguments applied for the augmented form (1.6). Since $\|w\| \leqslant]\|w\|\left[\lambda\right.$ for all $\lambda \in \mathbb{R}_{+}$, we see by (2.3), that the estimate (2.1) holds also for approximations calculated by (1.6).

Henceforth, we will use functional a posteriori error estimates for the Stokes problem derived in $[11,12]$. For a consequent exposition of the theory of functional a posteriori error estimates we refer the reader to $[8,10]$.

The following lemma is essential in deriving our main results. 
Lemma 2.1. Let $\Omega$ be a bounded domain with Lipschitz continuous boundary $\partial \Omega$. Then there exists a positive constant $C_{\mathrm{LBB}}$ depending on the domain $\Omega$ such that for any function $g \in \widetilde{L}_{2}(\Omega)$ there is a function $v \in V_{0}$ satisfying the condition $\operatorname{div} v=g$, and

$$
\|\nabla v\|_{\Sigma} \leqslant C_{\mathrm{LBB}}^{-1}\|g\| .
$$

Here $C_{\mathrm{LBB}}$ is the constant in the well-known Ladyzhenskaya-Babuška-Brezzi (LBB) condition (see, e.g., [1,2]). See proof in [6,7].

For some simple domains the constant $C_{\mathrm{LBB}}$, or the bounds for it, are known (see, e.g., $[3,5,9]$ ).

Lemma 2.1 implies an important corollary. Let $v \in V_{0}$, and $\operatorname{div} v=g$. Then there exists a function $v_{g} \in V_{0}$ such that $\operatorname{div}\left(v-v_{g}\right)=0$, and

$$
\left\|\nabla v_{g}\right\|_{\Sigma} \leqslant C_{\mathrm{LBB}}^{-1}\|g\|=C_{\mathrm{LBB}}^{-1}\|\operatorname{div} v\| .
$$

This means that there exists a solenoidal field $v_{0}=\left(v-v_{g}\right) \in \stackrel{\circ}{S}(\Omega)$ such that

$$
\left\|\nabla\left(v-v_{0}\right)\right\|_{\Sigma} \leqslant C_{\mathrm{LBB}}^{-1}\|\operatorname{div} v\| .
$$

A similar estimate holds for $v \in V_{0}+u_{D}$. Indeed, for $v-u_{D}$ we can find a solenoidal field $v_{0} \in \stackrel{S}{S}(\Omega)$ such that

$$
\left\|\nabla\left(v-u_{D}-v_{0}\right)\right\|_{\Sigma} \leqslant C_{\mathrm{LBB}}^{-1}\left\|\operatorname{div}\left(v-u_{D}\right)\right\| \leqslant C_{\mathrm{LBB}}^{-1}\|\operatorname{div} v\| .
$$

Thus, we can find a function $w_{0} \in \stackrel{\circ}{S}(\Omega)+u_{D}$ such that

$$
\left\|\nabla\left(v-w_{0}\right)\right\|_{\Sigma} \leqslant C_{\mathrm{LBB}}^{-1}\|\operatorname{div} v\| .
$$

With the help of (2.4) we can now derive our main results. We show that the errors of $u^{k}$ and $p^{k}$ generated on the iteration $k$ of the Uzawa algorithm are both estimated from above by the $L_{2}$-norm of the divergence of $u^{k}$ multiplied by a constant depending on $C_{\mathrm{LBB}}$. The proofs are based on the derivation of functional $a$ posteriori error estimates for the generalized Stokes problem as they are presented in [12].

Theorem 2.2. Let $u^{k}$ be the exact solution computed on the iteration $k$ of the Uzawa algorithm. Then, for solutions calculated by (1.5) or (1.6), we have

$$
\left\|u-u^{k}\right\| \leqslant 2 C\left\|\operatorname{div} u^{k}\right\|
$$

where

$$
C:=C_{\mathrm{LBB}}^{-1} \sqrt{C_{\mathrm{F}}^{2} \bar{\mu}+\bar{v}} .
$$

Here $C_{\mathrm{F}}$ is the constant in the Friedrichs inequality

$$
\|w\| \leqslant C_{\mathrm{F}}\|\nabla w\|_{\Sigma}
$$

and $C_{\mathrm{LBB}}$ is the constant in the $\mathrm{LBB}-$ condition. 
Proof. Let $u_{0} \in \stackrel{\circ}{S}(\Omega)+u_{D}$ be such that, by using (2.4), we have

$$
\left\|\nabla\left(u^{k}-u_{0}\right)\right\|_{\Sigma} \leqslant C_{\mathrm{LBB}}^{-1}\left\|\operatorname{div} u^{k}\right\| .
$$

Let the pair $\left(u^{k}, p^{k}\right)$ be an approximation of the saddle point computed on the iteration $k$. We can now write

$$
\left\|u-u^{k}\right\| \leqslant\left\|u-u_{0}\left|\|+\| u_{0}-u^{k} \|\right|\right.
$$

First, we estimate from above the first term on the right-hand side of (2.8). Let $w \in S^{\circ}$. By subtracting the integral $\int_{\Omega}\left(v \nabla u_{0}: \nabla w+\mu u_{0} \cdot w\right) \mathrm{d} x$ from both sides of (1.4) we obtain

$$
\begin{aligned}
& \int_{\Omega}\left(v \nabla\left(u-u_{0}\right): \nabla w+\mu\left(u-u_{0}\right)\right.\cdot w) \mathrm{d} x \\
&=\int_{\Omega}\left(\left(f-\mu u_{0}\right) \cdot w-v \nabla u_{0}: \nabla w\right) \mathrm{d} x .
\end{aligned}
$$

It is easy to see that

$$
\int_{\Omega}(\operatorname{Div} \tau \cdot w+\tau: \nabla w) \mathrm{d} x=0 \quad \forall \tau \in \Sigma(\operatorname{Div}, \Omega), w \in V_{0}(\Omega)
$$

and

$$
\int_{\Omega}(\nabla q \cdot w+q \operatorname{div} w) \mathrm{d} x=0 \quad \forall q \in W_{2}^{1}(\Omega, \mathbb{R}), w \in V_{0}(\Omega) . .
$$

By adding (2.10) and (2.11) to the right-hand side of (2.9), we rewrite it in the form

$$
\int_{\Omega}\left(\left(f-\mu u_{0}+\operatorname{Div} \tau-\nabla q\right) \cdot w+\left(\tau-v \nabla u_{0}\right): \nabla w\right) \mathrm{d} x
$$

which is equivalent to

$$
\begin{aligned}
\int_{\Omega}\left(\left(f-\mu u^{k}+\operatorname{Div} \tau\right.\right. & \left.-\nabla q) \cdot w+\left(\tau-v \nabla u^{k}\right): \nabla w\right) \mathrm{d} x \\
& +\int_{\Omega}\left(v \nabla\left(u^{k}-u_{0}\right): \nabla w+\mu\left(u^{k}-u_{0}\right) \cdot w\right) \mathrm{d} x .
\end{aligned}
$$

Let us choose $\tau=v \nabla u^{k}$ and $q=p^{k}$. In view of (1.5), we see that that the first integral of (2.13) vanishes. Indeed,

$$
\begin{aligned}
\int_{\Omega}\left(\left(f-\mu u^{k}+\operatorname{Div} v \nabla u^{k}-\nabla p^{k}\right) \cdot w+\left(v \nabla u^{k}-v \nabla u^{k}\right): \nabla w\right) \mathrm{d} x \\
=\int_{\Omega}\left(f \cdot w+p^{k} \operatorname{div} w-v \nabla u^{k}: \nabla w-\mu u^{k} \cdot w\right) \mathrm{d} x=0 .
\end{aligned}
$$


Since $w$ is a function from $\stackrel{\circ}{S}$, the same conclusion is also true if $u^{k}$ has been calculated by (1.6). We combine (2.9) with (2.12)-(2.14), and arrive at the relation

$$
\begin{aligned}
\int_{\Omega}\left(v \nabla\left(u-u_{0}\right): \nabla w\right. & \left.+v\left(u-u_{0}\right) \cdot w\right) \mathrm{d} x \\
& =\int_{\Omega}\left(v \nabla\left(u^{k}-u_{0}\right): \nabla w+\mu\left(u^{k}-u_{0}\right) \cdot w\right) \mathrm{d} x .
\end{aligned}
$$

The right-hand side of (2.15) can be estimated from above as follows:

$$
\begin{aligned}
\int_{\Omega}\left(v \nabla\left(u^{k}-u_{0}\right): \nabla w+\mu\left(u^{k}-u_{0}\right) \cdot w\right) \mathrm{d} x \\
\quad=\int_{\Omega}\left(\sqrt{v} \nabla\left(u^{k}-u_{0}\right): \sqrt{v} \nabla w+\sqrt{\mu}\left(u^{k}-u_{0}\right) \cdot \sqrt{\mu} w\right) \mathrm{d} x \\
\quad \leqslant\left\|\sqrt{v} \nabla\left(u^{k}-u_{0}\right)\right\|_{\Sigma}\|\sqrt{v} \nabla w\|_{\Sigma}+\left\|\sqrt{\mu}\left(u^{k}-u_{0}\right)\right\|\|\sqrt{\mu} w\| \\
\quad \leqslant\left\|u^{k}-u_{0}\right\| \mid\|w\|
\end{aligned}
$$

where we have used the Cauchy-Schwarz inequality. We set $w=u-u_{0}$, and find that

$$
\mid\left\|u-u_{0}\right\| \leqslant\left\|u^{k}-u_{0}\right\| .
$$

Note that for all $w \in V$ we have

$$
\begin{aligned}
\|w\|^{2} & =\|\sqrt{v} \nabla w\|_{\Sigma}^{2}+\|\sqrt{\mu} w\|^{2} \\
& \leqslant \bar{v}\|\nabla w\|_{\Sigma}^{2}+\bar{\mu}\|w\|^{2} \\
& \leqslant \bar{v}\|\nabla w\|_{\Sigma}^{2}+C_{\mathrm{F}}^{2} \bar{\mu}\|\nabla w\|_{\Sigma}^{2} \\
& \leqslant\left(C_{\mathrm{F}}^{2} \bar{\mu}+\bar{v}\right)\|\nabla w\|_{\Sigma}^{2} .
\end{aligned}
$$

We substitute (2.17) into (2.8), and use (2.18) with $w=u-u_{0}$, and obtain

$$
\begin{aligned}
\left\|u-u^{k}\right\| & \leqslant 2\left\|u_{0}-u^{k}\right\| \\
& \leqslant 2 \sqrt{C_{\mathrm{F}}^{2} \bar{\mu}+\bar{v}}\left\|\nabla\left(u_{0}-u^{k}\right)\right\|_{\Sigma} .
\end{aligned}
$$

Now, (2.7) and (2.19) imply the estimate

$$
\left\|u-u^{k}\right\| \leqslant 2 C_{\mathrm{LBB}}^{-1} \sqrt{C_{\mathrm{F}}^{2} \bar{\mu}+\bar{v}}\left\|\operatorname{div} u^{k}\right\|=2 C\left\|\operatorname{div} u^{k}\right\|
$$

where $C$ is defined in (2.6).

In order to prove a similar estimate for the pressure, we also need Lemma 2.1. Let $q \in \widetilde{L}_{2}$ be an approximation of the exact pressure $p$. Then $(p-q) \in \widetilde{L}_{2}$ and there exists a function $\bar{w} \in V_{0}$ such that

$$
\operatorname{div}(\bar{w})=p-q
$$


and

$$
\|\nabla \bar{w}\|_{\Sigma} \leqslant C_{\mathrm{LBB}}^{-1}\|p-q\| .
$$

Theorem 2.3. Let $p^{k}$ be the function computed on the iteration $k$ of the Uzawa algorithm. Then,

$$
\left\|p-p^{k}\right\| \leqslant \mathbb{C}\left\|\operatorname{div} u^{k}\right\|
$$

where $\mathbb{C}=2 C^{2}$ for (1.5), and $\mathbb{C}=2 C^{2}+\lambda$ for (1.6).

Proof. We use (2.20) for $q=p^{k}$ and obtain

$$
\left\|p-p^{k}\right\|^{2}=\int_{\Omega} \operatorname{div} \bar{w}\left(p-p^{k}\right) \mathrm{d} x=\int_{\Omega} \operatorname{div} \bar{w} p+\nabla p^{k} \cdot \bar{w} \mathrm{~d} x .
$$

Multiplying (1.1) by $\bar{w}$ and integrating over $\Omega$, we obtain

$$
\int_{\Omega} \operatorname{div} \bar{w} p \mathrm{~d} x=\int_{\Omega}(v \nabla u: \nabla \bar{w}+\mu u \cdot \bar{w}-f \cdot \bar{w}) \mathrm{d} x .
$$

In view of this relation, we have

$$
\left\|p-p^{k}\right\|^{2}=\int_{\Omega}\left(v \nabla u: \nabla \bar{w}+\mu u \cdot \bar{w}-f \cdot \bar{w}+\nabla p^{k} \cdot \bar{w}\right) \mathrm{d} x .
$$

We use (2.10) with $w=\bar{w}$, and arrive at the relation

$$
\begin{aligned}
\left\|p-p^{k}\right\|^{2}= & \int_{\Omega}\left(\left(-f+\mu u^{k}-\operatorname{Div} \tau+\nabla p^{k}\right) \cdot \bar{w}+\left(v \nabla u^{k}-\tau\right): \nabla \bar{w}\right) \mathrm{d} x \\
& +\int_{\Omega}\left(v \nabla\left(u-u^{k}\right): \nabla \bar{w}+\mu\left(u-u^{k}\right) \cdot \bar{w}\right) \mathrm{d} x
\end{aligned}
$$

As before, we choose $\tau=v \nabla u^{k}$, and observe that the first integral is zero. By estimating the latter integral with the help of the same arguments as in (2.16), we find that

$$
\left\|p-p^{k}\right\|^{2} \leqslant\left\|u-u^{k}\right\| \mid\|\bar{w}\| .
$$

By (2.18) and (2.21), we obtain

$$
\begin{aligned}
\|\bar{w}\|^{2} & \leqslant\left(C_{\mathrm{F}}^{2} \bar{\mu}+\bar{v}\right)\|\nabla \bar{w}\|_{\Sigma}^{2} \\
& \leqslant C_{\mathrm{LBB}}^{-2}\left(C_{\mathrm{F}}^{2} \bar{\mu}+\bar{v}\right)\left\|p-p^{k}\right\|^{2} \\
& =C^{2}\left\|p-p^{k}\right\|^{2}
\end{aligned}
$$

where $C$ is defined in (2.6). Substituting (2.25) into (2.24) results in the estimate

$$
\left\|p-p^{k}\right\| \leqslant C\left\|u-u^{k}\right\| .
$$

Now, we apply Theorem 2.2 and deduce (2.22). 
In the case of (1.6), we add

$$
\int_{\Omega} \lambda \operatorname{div}\left(u^{k}-u^{k}\right) \operatorname{div} \bar{w} \mathrm{~d} x=0
$$

to $(2.23)$ and obtain

$$
\begin{aligned}
\left\|p-p^{k}\right\|^{2}= & \int_{\Omega}\left(\left(-f+\mu u^{k}-\operatorname{Div} \tau+\nabla p^{k}\right) \cdot \bar{w}+\lambda \operatorname{div} u^{k} \operatorname{div} \bar{w}\right) \mathrm{d} x \\
& +\int_{\Omega}\left(v \nabla u^{k}-\tau\right): \nabla \bar{w} \mathrm{~d} x \\
& +\int_{\Omega}\left(v \nabla\left(u-u^{k}\right): \nabla \bar{w}+\mu\left(u-u^{k}\right) \cdot \bar{w}-\lambda \operatorname{div} u^{k} \operatorname{div} \bar{w}\right) \mathrm{d} x .
\end{aligned}
$$

Again, we choose $\tau=v \nabla u^{k}$, and see from (1.6) that the first and second integrals are zero. By estimating the latter integral with same arguments as in (2.16), we obtain

$$
\left\|p-p^{k}\right\|^{2} \leqslant\left\|u-u^{k}\right\|\|\| \bar{w}\|+\lambda\| \operatorname{div} u^{k}\|\| \operatorname{div} \bar{w} \| .
$$

Recall that $\operatorname{div} \bar{w}=p-p^{k}$. Now, (2.25) and (2.26) imply the estimate

$$
\left\|p-p^{k}\right\| \leqslant C\left\|u-u^{k}\right\| \mid+\lambda\left\|\operatorname{div} u^{k}\right\| .
$$

Applying Theorem 2.2 results in (2.22).

By Theorems 2.2 and 2.3, we easily conclude the following statement.

Remark 2.1. The classical Stokes problem corresponds to the case where $\mu \equiv 0$ and $v$ is a constant. Let $\left(u^{k}, p^{k}\right)$ be the exact solution computed on the iteration $k$ of the Uzawa algorithm, for the Stokes problem. Then, for velocity we have (for both cases (1.5) and (1.6))

$$
\left\|\nabla\left(u-u^{k}\right)\right\| \leqslant 2 C_{\mathrm{LBB}}^{-1}\left\|\operatorname{div} u^{k}\right\| .
$$

For the pressure we have

$$
\left\|p-p^{k}\right\| \leqslant \tilde{\mathbb{C}}\left\|\operatorname{div} u^{k}\right\|
$$

where $\tilde{\mathbb{C}}=2 C_{\mathrm{LBB}}^{-2} v$ for (1.5) and $\tilde{\mathbb{C}}=2 C_{\mathrm{LBB}}^{-2} \nu+\lambda$ for (1.6).

\section{Computable error estimates for approximations generated by the Uzawa algorithm}

Let $\mathscr{T}_{h}$ be a mesh having the characteristic size $h$, and let the spaces $V_{0 h}\left(\Omega, \mathbb{R}^{n}\right)$ and $Q_{h}(\Omega)$ be finite dimensional subspaces of $V_{0}\left(\Omega, \mathbb{R}^{n}\right)$ and $\widetilde{L}_{2}(\Omega)$, respectively. We assume that for all $v_{h} \in V_{0 h}+u_{D}$ it holds that $\operatorname{div} v_{h} \in Q_{h}$. We also assume that the 
spaces are constructed so that they satisfy the discrete LBB-condition, i.e, for any $q_{h} \in Q_{h}$ with zero mean, there exists $v_{h} \in V_{0 h}$ such that

$$
\operatorname{div} v_{h}=q_{h}
$$

and

$$
\left\|\nabla v_{h}\right\|_{\Sigma} \leqslant c\left\|q_{h}\right\|
$$

where the positive constant $c$ does not depend on $h$.

Let $u_{h}^{k} \in V_{0 h}+u_{D}$ be an approximation of $u^{k}$ calculated on the mesh $\mathscr{T}_{h}$. We need to combine the error of the pure Uzawa algorithm with the approximation error. Below we present the corresponding results, where we set $p^{k}=p_{h}^{k} \in Q_{h}$ on the iteration $k$, and understand $u^{k}$ as satisfying (1.5), or (1.6), with the chosen $p_{h}^{k}$. Then, the pair $\left(u^{k}, p_{h}^{k}\right)$ can be viewed as the exact pair associated with the Uzawa algorithm on iteration $k$.

Our first goal is to derive fully computable error majorants $M_{\oplus}^{k}$ and $M_{\oplus}^{k, \lambda}$ for approximate solutions (e.g., $u_{h}^{k}$ ) of the problems generated at the first step of Uzawa algorithm by the Lagrangians $L$ and $L_{A}$, respectively. In order to make the quality of the majorants robust with respect to small or large values of the material functions $v$ or $\mu$, we apply the same method that was suggested in [12] for the generalized Stokes problem.

Later we combine these estimates with the estimates of the difference between $u$ and $u^{k}$ and obtain estimates applicable for approximate solutions computed within the framework of finite dimensional approximations.

First, we prove the following result for the problem generated by the Lagrangian $L$.

Theorem 3.1. Let $\left(u^{k}, p_{h}^{k}\right)$ be the exact solution on the iteration $k$ of the Uzawa algorithm. Then, for the solutions calculated by (1.5), and for an approximation $u_{h}^{k} \in V_{0 h}+u_{D}$ we have

$$
\left\|u^{k}-u_{h}^{k}\right\|^{2} \leqslant M_{\oplus}^{k}\left(u_{h}^{k}, p_{h}^{k}, \tau, \beta\right) \quad \forall \tau \in H(\operatorname{Div}, \Omega), \beta \in \mathbb{R}_{+}
$$

where

$$
M_{\oplus}^{k}\left(u_{h}^{k}, p_{h}^{k}, \tau, \beta\right):=\int_{\Omega} H_{1}(v, \mu, \beta) r^{2}\left(u_{h}^{k}, \tau\right) \mathrm{d} x+H_{2}(\beta)\left\|\sqrt{v}^{-1} d\left(u_{h}^{k}, p_{h}^{k}, \tau\right)\right\|_{\Sigma}^{2}
$$

and

$$
\begin{aligned}
H_{1}(v, \mu, \beta) & :=\frac{C_{\mathrm{F}}^{2}(1+\beta)}{\underline{v}+C_{\mathrm{F}}^{2}(1+\beta) \mu} \\
H_{2}(\beta) & :=1+\beta^{-1} \\
r\left(u_{h}^{k}, \tau\right) & :=f-\mu u_{h}^{k}+\operatorname{Div} \tau \\
d\left(u_{h}^{k}, p_{h}^{k}, \tau\right) & :=\tau-v \nabla u_{h}^{k}+\mathbb{I} p_{h}^{k} .
\end{aligned}
$$

Here II denotes the unit tensor. 
Proof. By equation (1.5) we have

$$
\int_{\Omega}\left(v \nabla u^{k}: \nabla w+\mu u^{k} \cdot w\right) \mathrm{d} x=\int_{\Omega}\left(f \cdot w+p_{h}^{k} \operatorname{div} w\right) \mathrm{d} x .
$$

We subtract the integral $\int_{\Omega}\left(v \nabla u_{h}^{k}: \nabla w+\mu u_{h}^{k} \cdot w\right) \mathrm{d} x$ from both sides of the above equation, and obtain

$$
\begin{aligned}
\int_{\Omega} v \nabla\left(u^{k}-u_{h}^{k}\right): \nabla w & +\mu\left(u^{k}-u_{h}^{k}\right) \cdot w \mathrm{~d} x \\
& =\int_{\Omega}\left(\left(f-\mu u_{h}^{k}\right) \cdot w-v \nabla u_{h}^{k}: \nabla w+p_{h}^{k} \operatorname{div} w\right) \mathrm{d} x .
\end{aligned}
$$

By adding (2.10) to the right-hand side of (3.5) we have

$$
\begin{aligned}
\int_{\Omega} & \left(v \nabla\left(u^{k}-u_{h}^{k}\right): \nabla w+\mu\left(u^{k}-u_{h}^{k}\right) \cdot w\right) \mathrm{d} x \\
\quad= & \int_{\Omega}\left(\left(f-\mu u_{h}^{k}+\operatorname{Div} \tau\right) \cdot w+\left(\tau-v \nabla u_{h}^{k}+\mathbb{I} p_{h}^{k}\right): \nabla w\right) \mathrm{d} x \\
\quad= & \int_{\Omega}\left(r\left(u_{h}^{k}, \tau\right) \cdot w+d\left(u_{h}^{k}, p_{h}^{k}, \tau\right): \nabla w\right) \mathrm{d} x
\end{aligned}
$$

where we have used the notation (3.3) and (3.4). Note that

$$
\begin{aligned}
\int_{\Omega} r \cdot w \mathrm{~d} x & =\int_{\Omega}\left(\sqrt{\mu}^{-1} \alpha r \cdot \sqrt{\mu} w+(1-\alpha) r \cdot w\right) \mathrm{d} x \\
& \leqslant\left\|\sqrt{\mu}^{-1} \alpha r\right\|\|\sqrt{\mu} w\|+\|(1-\alpha) r\|\|w\| \\
& \leqslant\left\|\sqrt{\mu}^{-1} \alpha r\right\|\|\sqrt{\mu} w\|+C_{\mathrm{F}} \sqrt{\underline{v}}^{-1}\|(1-\alpha) r\|\|\sqrt{v} \nabla w\|_{\Sigma}
\end{aligned}
$$

where $0 \leqslant \alpha(x) \leqslant 1$. Also, we have

$$
\int_{\Omega} d: \nabla w \mathrm{~d} x \leqslant\left\|\sqrt{v}^{-1} d\right\|_{\Sigma}\|\sqrt{v} \nabla w\|_{\Sigma}
$$

By (3.7) and (3.8) the right-hand side of (3.6) becomes

$$
\begin{aligned}
& \left(C_{\mathrm{F}} \sqrt{\underline{v}}^{-1}\|(1-\alpha) r\|+\left\|\sqrt{v}^{-1} d\right\|_{\Sigma}\right)\|\sqrt{v} \nabla w\|_{\Sigma}+\left\|\sqrt{\mu}^{-1} \alpha r\right\|\|\sqrt{\mu} w\| \\
& \quad \leqslant \sqrt{\left(C_{\mathrm{F}} \sqrt{\underline{v}}^{-1}\|(1-\alpha) r\|+\left\|\sqrt{v}^{-1} d\right\|_{\Sigma}\right)^{2}+\left\|\sqrt{\mu}^{-1} \alpha r\right\|^{2}}\|w\| .
\end{aligned}
$$

We set $w=u^{k}-u_{h}^{k}$, use (3.6) and (3.9), and obtain

$$
\begin{aligned}
\left\|u^{k}-u_{h}^{k}\right\|^{2} \leqslant & \left(C_{\mathrm{F}} \sqrt{\underline{v}}^{-1}\|(1-\alpha) r\|+\left\|\sqrt{v}^{-1} d\right\|_{\Sigma}\right)^{2}+\left\|\sqrt{\mu}^{-1} \alpha r\right\|^{2} \\
\leqslant & (1+\beta) C_{\mathrm{F}}^{2} \underline{v}^{-1}\|(1-\alpha) r\|^{2} \\
& +\left(1+\beta^{-1}\right)\left\|\sqrt{v}^{-1} d\right\|_{\Sigma}^{2}+\left\|\sqrt{\mu}^{-1} \alpha r\right\|^{2} .
\end{aligned}
$$


It is easy to see that the optimal value of $\alpha$ is defined by the relation

$$
\alpha=\frac{C_{\mathrm{F}}^{2}(1+\beta) \mu}{\underline{v}+C_{\mathrm{F}}^{2}(1+\beta) \mu}
$$

so that (3.10) implies the estimate

$$
\begin{aligned}
\left\|u^{k}-u_{h}^{k}\right\|^{2} & \leqslant \int_{\Omega} \frac{C_{\mathrm{F}}^{2}(1+\beta)}{\underline{v}+C_{\mathrm{F}}^{2}(1+\beta) \mu} r^{2} \mathrm{~d} x+\left(1+\beta^{-1}\right)\left\|\sqrt{v}^{-1} d\right\|_{\Sigma}^{2} \\
& =\int_{\Omega} H_{1} r^{2} \mathrm{~d} x+H_{2}\left\|\sqrt{v}^{-1} d\right\|_{\Sigma}^{2}
\end{aligned}
$$

where we have used the notation (3.1) and (3.2).

Remark 3.1. It is easy to see that the upper bound $M_{\oplus}^{k}$ is sharp. Indeed, by setting $\tau=v \nabla u^{k}-\mathbb{I} p_{h}^{k}$, and letting $\beta$ tend to infinity, we get the exact error in the energy norm $\|\cdot\| \cdot \|$.

A similar estimate can be derived for the problem generated by the augmented Lagrangian $L_{A}$.

Theorem 3.2. Let $\left(u^{k}, p_{h}^{k}\right)$ be the exact solution on the iteration $k$ of the Uzawa algorithm. Then, for the solutions calculated by (1.6), and for an approximation $u_{h}^{k} \in V_{0 h}+u_{D}$ we have

$$
\left.\left\|u^{k}-u_{h}^{k}\right\|^{2} \leqslant\right]\left\|u^{k}-u_{h}^{k}\right\|_{\lambda}^{2} \leqslant M_{\oplus}^{k, \lambda}\left(u_{h}^{k}, p_{h}^{k}, \tau, \beta\right) \quad \forall \tau \in H(\operatorname{Div}, \Omega), \beta \in \mathbb{R}_{+}
$$

where

$$
M_{\oplus}^{k, \lambda}\left(u_{h}^{k}, p_{h}^{k}, \tau, \beta\right):=\int_{\Omega} H_{1}(v, \mu, \beta) r^{2}\left(u_{h}^{k}, \tau\right) \mathrm{d} x+H_{2}(\beta)\left\|\sqrt{v}^{-1} d^{\lambda}\left(u_{h}^{k}, p_{h}^{k}, \tau\right)\right\|_{\Sigma}^{2} .
$$

The quantities $H_{1}, H_{2}$, and $r$ are defined in (3.1)-(3.3), and

$$
d^{\lambda}\left(u_{h}^{k}, p_{h}^{k}, \tau\right):=\tau-v \nabla u_{h}^{k}+\mathbb{I}\left(p_{h}^{k}-\lambda \operatorname{div} u_{h}^{k}\right) .
$$

Proof. By (1.6), we have

$$
\int_{\Omega}\left(v \nabla u^{k}: \nabla w+\mu u^{k} \cdot w+\lambda \operatorname{div} u^{k} \operatorname{div} w\right) \mathrm{d} x=\int_{\Omega}\left(f \cdot w+p_{h}^{k} \operatorname{div} w\right) \mathrm{d} x .
$$

We subtract the integral $\int_{\Omega}\left(v \nabla u_{h}^{k}: \nabla w+\mu u_{h}^{k} \cdot w+\lambda \operatorname{div} u_{h}^{k} \operatorname{div} w\right) \mathrm{d} x$ from both sides 
of the above equation, and use (2.10), and obtain

$$
\begin{aligned}
\int_{\Omega} & \left(v \nabla\left(u^{k}-u_{h}^{k}\right): \nabla w+\mu\left(u^{k}-u_{h}^{k}\right) \cdot w+\lambda \operatorname{div}\left(u^{k}-u_{h}^{k}\right) \operatorname{div} w\right) \mathrm{d} x \\
& =\int_{\Omega}\left(\left(f-\mu u_{h}^{k}\right) \cdot w-v \nabla u_{h}^{k}: \nabla w+\left(p_{h}^{k}-\lambda \operatorname{div} u_{h}^{k}\right) \operatorname{div} w\right) \mathrm{d} x \\
& =\int_{\Omega}\left(\left(f-\mu u_{h}^{k}+\operatorname{Div} \tau\right) \cdot w+\left(\tau-v \nabla u_{h}^{k}+\mathbb{I}\left(p_{h}^{k}-\lambda \operatorname{div} u_{h}^{k}\right)\right): \nabla w\right) \mathrm{d} x \\
& =\int_{\Omega}\left(r\left(u_{h}^{k}, \tau\right) \cdot w+d^{\lambda}\left(u_{h}^{k}, p_{h}^{k}, \tau\right): \nabla w\right) \mathrm{d} x
\end{aligned}
$$

where we have used the notation (3.3) and (3.12). By the same arguments as in (3.7) and (3.8), we represent the right-hand side of (3.13) in the form

$$
\begin{aligned}
& \left(C_{\mathrm{F}} \sqrt{\underline{v}}^{-1}\|(1-\alpha) r\|+\left\|\sqrt{v}^{-1} d^{\lambda}\right\|_{\Sigma}\right)\|\sqrt{v} \nabla w\|_{\Sigma}+\left\|\sqrt{\mu}^{-1} \alpha r\right\|\|\sqrt{\mu} w\| \\
& \leqslant \sqrt{\left.\left(C_{\mathrm{F}} \sqrt{\underline{v}}^{-1}\|(1-\alpha) r\|+\left\|\sqrt{v}^{-1} d^{\lambda}\right\|_{\Sigma}\right)^{2}+\left\|\sqrt{\mu}^{-1} \alpha r\right\|^{2}\right]\|w\|[\lambda}
\end{aligned}
$$

since $\|w\| \leqslant \leqslant\|w\|\left[\lambda\right.$. By choosing $w=u^{k}-u_{h}^{k}$, (3.13) and (3.14) give

$$
\begin{aligned}
]\left.\left\|u^{k}-u_{h}^{k}\right\|\right|_{\lambda} ^{2} \leqslant & \left(C_{\mathrm{F}} \sqrt{\underline{v}}^{-1}\|(1-\alpha) r\|+\left\|\sqrt{v}^{-1} d^{\lambda}\right\|_{\Sigma}\right)^{2}+\left\|\sqrt{\mu}^{-1} \alpha r\right\|^{2} \\
\leqslant & (1+\beta) C_{\mathrm{F}}^{2} \underline{v}^{-1}\|(1-\alpha) r\|^{2} \\
& +\left(1+\beta^{-1}\right)\left\|\sqrt{v}^{-1} d^{\lambda}\right\|_{\Sigma}^{2}+\left\|\sqrt{\mu}^{-1} \alpha r\right\|^{2} .
\end{aligned}
$$

Again, we see that the optimal value of $\alpha$ is given by the relation (3.11), and obtain

$$
\begin{aligned}
\left\|u^{k}-u_{h}^{k}\right\| \|_{\lambda}^{2} & \leqslant \int_{\Omega} \frac{C_{\mathrm{F}}^{2}(1+\beta)}{\underline{v}+C_{\mathrm{F}}^{2}(1+\beta) \mu} r^{2} \mathrm{~d} x+\left(1+\beta^{-1}\right)\left\|\sqrt{v}^{-1} d^{\lambda}\right\|_{\Sigma}^{2} \\
& =\int_{\Omega} H_{1} r^{2} \mathrm{~d} x+H_{2}\left\|\sqrt{v}^{-1} d^{\lambda}\right\|_{\Sigma}^{2}
\end{aligned}
$$

where we have used the notation (3.1) and (3.2).

Finally, by using Theorems 2.2, 3.1, and 3.2 we obtain the final result.

Theorem 3.3. Let $u$ be the exact velocity, $\left(u^{k}, p_{h}^{k}\right)$ be the exact solution calculated on the iteration $k$ of the Uzawa algorithm, and $u_{h}^{k} \in V_{0 h}+u_{D}$ be an approximation of the velocity calculated on this iteration. For (1.5) we have

$$
\left\|u-u_{h}^{k}\right\| \leqslant \mathbf{M}_{\oplus}^{k}\left(u_{h}^{k}, p_{h}^{k}, \tau, \beta\right) \quad \forall \tau \in H(\operatorname{Div}, \Omega), \beta \in \mathbb{R}_{+}
$$

and for (1.6) we have

$$
\left\|u-u_{h}^{k}\right\| \leqslant \mathbf{M}_{\oplus}^{k, \lambda}\left(u_{h}^{k}, p_{h}^{k}, \tau, \beta\right) \quad \forall \tau \in H(\operatorname{Div}, \Omega), \beta \in \mathbb{R}_{+}
$$


where

$$
\begin{aligned}
\mathbf{M}_{\oplus}^{k}\left(u_{h}^{k}, p_{h}^{k}, \tau, \beta\right) & :=2 C\left\|\operatorname{div} u_{h}^{k}\right\|+\left(2 C \sqrt{\underline{v}}^{-1}+1\right) \sqrt{M_{\oplus}^{k}\left(u_{h}^{k}, p_{h}^{k}, \tau, \beta\right)} \\
\mathbf{M}_{\oplus}^{k, \lambda}\left(u_{h}^{k}, p_{h}^{k}, \tau, \beta\right) & :=2 C\left\|\operatorname{div} u_{h}^{k}\right\|+\left(2 C \sqrt{\underline{v}}^{-1}+1\right) \sqrt{M_{\oplus}^{k, \lambda}\left(u_{h}^{k}, p_{h}^{k}, \tau, \beta\right)}
\end{aligned}
$$

with $C$ defined in (2.6).

Proof. It is clear that

$$
\left\|u-u_{h}^{k}\right\|\left|\leqslant\left\|u-u^{k}\right\|+\left\|u^{k}-u_{h}^{k}\right\|\right| .
$$

By Theorem 2.2 we have

$$
\begin{aligned}
\left\|u-u_{h}^{k}\right\| & \leqslant 2 C\left\|\operatorname{div} u^{k}\right\|+\left\|u^{k}-u_{h}^{k}\right\| \\
& \leqslant 2 C\left\|\operatorname{div} u_{h}^{k}\right\|+2 C\left\|\operatorname{div}\left(u^{k}-u_{h}^{k}\right)\right\|+\left\|u^{k}-u_{h}^{k}\right\| \\
& \leqslant 2 C\left\|\operatorname{div} u_{h}^{k}\right\|+2 C \sqrt{\underline{v}}^{-1}\left\|\sqrt{v} \nabla\left(u^{k}-u_{h}^{k}\right)\right\|+\left\|u^{k}-u_{h}^{k}\right\| \\
& \leqslant 2 C\left\|\operatorname{div} u_{h}^{k}\right\|+\left(2 C \sqrt{\underline{v}}^{-1}+1\right)\left\|u^{k}-u_{h}^{k}\right\| .
\end{aligned}
$$

Using the upper bounds presented in Theorems 3.1 and 3.2 for the two cases (1.5) and (1.6), respectively, we arrive at the result.

Finally, we note that estimates for the pressure follows from the above derived estimates. The exact pressure in the Uzawa algorithm is calculated by (1.7), i.e.,

$$
p^{k+1}=\left(p_{h}^{k}-\rho \operatorname{div} u^{k}\right) \in \widetilde{L}_{2}(\Omega)
$$

and an approximation of it is calculated within the framework of the selected finite dimensional subspaces, i.e.,

$$
p_{h}^{k+1}=\left(p_{h}^{k}-\rho \operatorname{div} u_{h}^{k}\right) \in Q_{h}(\Omega) .
$$

Theorem 3.4. Let $\left(u^{k}, p_{h}^{k}\right)$ be the exact solution calculated on the iteration $k$ of the Uzawa algorithm, and $u_{h}^{k} \in V_{0 h}+u_{D}$ be an approximation of the velocity calculated on this iteration. Now, we apply the estimates presented in Theorems 3.1 and 3.2, and obtain for (1.5):

$$
\left\|p^{k+1}-p_{h}^{k+1}\right\| \leqslant \rho \underline{\underline{v}}^{-1} \sqrt{M_{\oplus}^{k}\left(u_{h}^{k}, p_{h}^{k}, \tau, \beta\right)} \quad \forall \tau \in H(\operatorname{Div}, \Omega), \beta \in \mathbb{R}_{+}
$$

and for (1.6)

$$
\left\|p^{k+1}-p_{h}^{k+1}\right\| \leqslant \rho \sqrt{\underline{v}}^{-1} \sqrt{M_{\oplus}^{k, \lambda}\left(u_{h}^{k}, p_{h}^{k}, \tau, \beta\right)} \quad \forall \tau \in H(\operatorname{Div}, \Omega), \quad \beta \in \mathbb{R}_{+} .
$$


Proof. Indeed, from (3.15) and (3.16) we find that

$$
\begin{aligned}
\left\|p^{k+1}-p_{h}^{k+1}\right\| & =\rho\left\|\operatorname{div}\left(u^{k}-u_{h}^{k}\right)\right\| \\
& \leqslant \rho \sqrt{\underline{v}}^{-1}\left\|\sqrt{v} \nabla\left(u^{k}-u_{h}^{k}\right)\right\| \\
& \leqslant \rho \sqrt{\underline{v}}^{-1}\left\|u^{k}-u_{h}^{k}\right\| .
\end{aligned}
$$

Applying the error bounds presented in Theorems 3.1 and 3.2 completes the proof.

This paper is focused on theoretical analysis of a posteriori error bounds for approximations computed by the Uzawa algorithm. However, it is worth adding some comments on the practical applications of the above derived error majorants. The majorants contain the function $\tau \in H(\operatorname{Div}, \Omega)$ and a positive parameter $\beta$, which in general can be taken arbitrary. Getting sharp estimates requires a proper selection of them. Finding an optimal $\beta$ leads to a one-dimensional optimization problem which is easy solvable. The reconstruction of the stress tensor $\tau$ based upon computed functions $u_{h}^{k}$ and $p_{h}^{k}$ provides a reasonable first guess. A better selection can be performed by methods that have been developed and tested for various elliptic problems (see, e.g., $[8,10,14]$ and the references cited therein). A systematical study of computational questions in the context of above derived estimates will be exposed in a separate paper, which is now in preparation.

\section{References}

1. I. Babuška, The finite element method with Lagrangian multipliers, Numer. Math. (1973) 20, 179-192

2. F. Brezzi, On the existence, uniqueness and approximation of saddle-point problems arising from Lagrangian multipliers, RAIRO Sér. Rouge Snal. Numér (1974) 8(R-2), 129-151.

3. M. Dobrowolski, On the LBB condition in the numerical analysis of the Stokes equations, Appl. Numer. Math. (2005) 54, 314-323.

4. M. Fortin and R. Glowinski, Augmented Lagrangian methods: applications to the numerical solution of boundary-value problems, North-Holland, New-York, 1983.

5. C. O. Horgan and L. E. Payne, On inequalities of Korn, Friedrichs and Babuška-Aziz. Arch. Ration. Mech. Anal. (1982) 82, No. 2, 165-179.

6. O. A. Ladyzhenskaya, The Mathematical Theory of Viscous Incompressible Flow. Gordon and Breach, New York, 1969.

7. O. A. Ladyzhenskaya and V. A. Solonnikov, Some problems of vector analysis and generalized formulations of boundary value problems for the Navier-Stokes equation. Zap. Nauchn. Semin. LOMI (1976) 59, 81-116 (in Russian).

8. P. Neittaanmäki and S. Repin, Reliable Methods for Computer Simulation. Error Control and A Posteriori Estimates. Elsevier, Amsterdam, 2004.

9. M. A. Ol'shanskii and E. V. Chizhonkov, On the domain geometry dependence of the LBB condition. M2AN Math. Model. Numer. Anal. (2000) 34, No. 5, 935-951.

10. S. Repin, A Posteriori Estimates for Partial Differential Equations. Walter de Gruyter, Berlin, 2008. 
11. S. Repin, A posteriori estimates for the Stokes problem, J. Math. Sci. (2002) 109, No. 5, 19501964.

12. S. Repin and R. Stenberg, A posteriori error estimates for the generalized Stokes problem, $J$. Math. Sci. (2007) 142, No. 1, 1828-1843.

13. R. Temam, Navier-Stokes Equations. Theory and Numerical Analysis. North-Holland, New York, 1977.

14. J. Valdman, Minimization of functional majorant in a posteriori error analysis based on $H$ (div) multigrid-preconditioned CG method. Adv. Num. Anal. 2009, Article ID 164519. 